COMO CITAR ESTE ARTÍCULO:

Peña-Huertas, R., Ternera-Barrios, F. y RuizGonzález, L.E. (2019). Baldíos, teorías de la propiedad y altas cortes en Colombia. Revista Jurídicas, 16 (1), 28-41.

DOI: 10.17151/jurid.2019.16.1.3.

Recibido el 10 de julio de 2018

Aprobado el 26 de octubre de 2018

\section{Baldíos, teorías de la propiedad y altas Cortes en Colombia*}

\author{
Rocío Del Pilar Peña-Huertas ** \\ Francisco Ternera-Barrios *** \\ Luis EnRiQue RuIZ-GonZÁlez ****
}

\section{RESUMEN}

Este texto busca identificar las teorías sobre los derechos de propiedad que están involucradas en las providencias de las Cortes Constitucional y Suprema de Justicia en el tema de la asignación de derechos de propiedad sobre predios baldíos, evidenciando sus tensiones e interacciones recientes. Entonces, se asocian las teorías del utilitarismo y de Locke explicadas en un primer momento, con los argumentos revisados en 17 providencias de las Cortes referidas a acciones de tutela que pretenden revisar procesos de prescripción del dominio entre julio de 2014 y octubre de 2016. La revisión muestra los argumentos de la Corte Constitucional con la teoría utilitarista que fue respaldada por la Corte Suprema, excepto a inicios de 2016 cuando se acogieron nociones de la teoría de Locke sobre los derechos de propiedad. Además, se muestran intentos de abstraer la discusión sobre baldíos del ámbito constitucional para trasladarla al habitual escenario del derecho civil.
Palabras clave: derechos de propiedad, baldíos, Corte Constitucional, Corte Suprema de Justicia.

\footnotetext{
Esta investigación se enmarca en el proyecto Diseños Institucionales de la Regulación de Derechos de Propiedad Agraria en Colombia, que hace parte del programa red Observatorio de Restitución y Regulación de Derechos de Propiedad Agraria, financiado por Colciencias.

** Doctora en Derecho de la Universidad del Rosario. Docente de carrera académica de la misma universidad. Bogotá, Colombia. E-mail: rocio.pena@urosario.edu. co. Google Scholar. ORCID: 0000-0002-2167-8676 *** Doctor en Derecho de la Universidad Alfonso X el Sabio. Profesor titular de la Universidad del Rosario. Bogotá, Colombia. E-mail: francisco.ternera@urosario. edu.co. Google Scholar. ORCID: 0000-0002-7677-5692 **** Politólogo y abogado de la Universidad del Rosario. Investigador del Observatorio de Restitución y Regulación de Derechos de Propiedad Agraria. Bogotá, Colombia. E-mail: luise.ruiz@urosario.edu. co. Google Scholar. ORCID: 0000-0002-9397-6373
} 


\section{Vacant lots, theories of property and high Courts in Colombia}

\section{ABSTRACT}

This text aims to identify the theories of property rights that are involved in the Constitutional Court and the Supreme Court rulings on the issue of the allocation of private property rights on vacant lots showing their tensions and recent interactions. Then, the theories of utilitarianism and Locke's theories initially explained are associated with the arguments reviewed in 17 judicial decisions of the Constitutional Court and the Supreme Court referred to writs of amparo between July 2014 and October 2016. The review shows the arguments of the Constitutional Court with the utilitarian theory that was supported by the Supreme Court, except in early 2016 when notions of Locke's theory about property rights were accepted. In addition, attempts to abstract the discussion on vacant lots from the constitutional sphere to move it to the usual civil law scenario are being made.

KEY WORDS: property rights, vacant lots, Constitutional Court, Supreme Court of Justice. 


\section{Introducción}

El debate sobre los derechos de propiedad ha ocupado recientemente un lugar central en Colombia y hay múltiples manifestaciones de esta discusión en curso. En primer lugar, la implementación de la Ley de Víctimas y Restitución de Tierras desde 2011 ha propuesto la restitución de los derechos sobre la tierra de las víctimas del conflicto armado. El diseño del proceso de restitución incluyó innovaciones dirigidas a hacer efectiva la posibilidad de restituir esos derechos que en Colombia están afectados por la precariedad y la informalidad. Tal diseño no ha estado exento de resistencias y debates en torno a la legitimidad de las transacciones sobre la propiedad que se dieron en el contexto del conflicto armado y la figura de la buena fe exenta de culpa ha sido un aspecto central para considerar esa propiedad legítima o ilegítima Quinche-Ramírez, Peña-Huertas, Parada-Hernández, Ruiz-González y Álvarez-Morales (2015).

En segundo lugar, luego de varios intentos legislativos, el Estado colombiano adoptó la Ley 1776 de 2016 que entre su articulado propone la destinación de inmuebles baldíos a proyectos productivos que involucren a pequeños y medianos productores. Para algunos, estas disposiciones modifican sustancialmente la destinación privilegiada que han tenido los baldíos de la nación para ser adjudicados a la población campesina.

En tercer lugar, las negociaciones entre el gobierno nacional y la guerrilla de las FARC ha resultado en un acuerdo que, en su primer punto sobre reforma rural integral, involucra varios esfuerzos por regular la propiedad en Colombia, como la constitución de un fondo de tierras para distribuirlas a los campesinos o el programa de formalización masiva de la propiedad a pequeños y medianos productores del campo, junto con esfuerzos técnicos para que el Estado pueda regular la propiedad como el catastro multipropósito y el inventario de predios baldíos para saber su ubicación y estado de ocupación.

Por último, se han planteado iniciativas legislativas, actualmente en trámite que intentan responder a algunos propietarios de los Llanos Orientales del país y su deseo de acceder a títulos de propiedad sobre predios para los cuales está en discusión su naturaleza baldía o privada. Esto ha desatado una discusión con agencias del Estado interesadas en conservar la propiedad a favor de la nación para distribuirla en forma más equitativa.

Si bien este recuento muestra que desde hace por lo menos cinco años los derechos de propiedad están en las prioridades de la agenda pública del país, este artículo se enfoca en esta última discusión sobre la distinción entre propiedad baldía y propiedad privada, y en cómo tal discusión ha sido trasladada al ámbito judicial.

Mediante acciones de tutela que pretenden anular sentencias de prescripción sobre presuntos bienes baldíos, personas particulares y las agencias del Estado 
han conducido a un ámbito relevante de análisis sobre la constitucionalización del derecho privado pues un aspecto muy característico del derecho privado, como es el derecho a la propiedad privada, se traslada a escenarios y contextos constitucionales que, a su vez, impactan en transformaciones sobre las teorías de la propiedad que predominan en las jurisdicciones civiles y constitucionales. De ese modo, las teorías sobre la propiedad que se involucran en las decisiones judiciales sobre la distinción entre propiedad privada o baldía constituyen el otro ámbito relevante de análisis de este texto.

En Colombia se cuentan con trabajos importantes, punto de partida sobre las teorías sobre los derechos de propiedad que predominan en Colombia y en el derecho continental. Tal es el caso de los trabajos de Rengifo-Gardeazábal (2011) y Céspedes-Báez, Peña-Huertas, Cabana-González y Zuleta-Ríos (2015). Este trabajo, sin tener un carácter exhaustivo acerca del sustento teórico de las Cortes colombianas, pretende avanzar en la identificación de las teorías de derecho de propiedad que se asocian a determinadas decisiones judiciales en torno a la posibilidad de acceder a la propiedad de predios baldíos. Consideramos que el avance en este aspecto abre la puerta a un ejercicio sistemático de las diversas teorías de los derechos de propiedad, y su presencia y uso que de ellas hacen los jueces y las Cortes en Colombia'.

De este modo, este análisis se realiza al identificar algunas de las teorías de derechos de propiedad involucradas en sentencias proferidas entre 2014 y octubre de 2016 por la CSJ en sede de tutela, sentencias de casación, y sentencias de la Corte Constitucional en sede de revisión de tutela junto con algunos autos de seguimiento que abordan el tema de la propiedad sobre bienes baldíos de la nación. Para este trabajo se examinaron 17 sentencias proferidas por la Corte Constitucional (2) y la Corte Suprema de Justicia (15) entre julio de 2014 y agosto de 2016, en donde un asunto abordado es la cuestión de la administración de las tierras baldías de la nación y el impacto que tienen los procesos civiles de pertenencia sobre esa administración. Para esto, las Cortes parten de dos conceptos específicos: si el bien es un baldío, la propiedad se adquiere mediante la adjudicación; si el bien es privado, es posible adquirirlo por usucapión. El problema jurídico de las decisiones requiere necesariamente determinar la naturaleza del predio y cuáles son los elementos indicativos de esa naturaleza. De cada providencia se analizó cuál definición de baldío utiliza en su fundamentación y cuáles fueron las cuestiones de hecho que llevaron a cada autoridad judicial a considerar que estaba ante un bien de esa naturaleza. Toda esta información fue consignada en una matriz para sistematizar esta información².

\footnotetext{
1 En esa dirección podría discutirse la persistencia de teorías usuales en el ámbito del derecho continental como las llamadas teorías subjetiva y objetiva de la propiedad. Empero, este aspecto trasciende los propósitos de este artículo. Agradecemos a uno de los pares evaluadores del artículo por esta contribución.

${ }^{2}$ Esta matriz puede ser consultada en: http://www.observatoriodetierras.org/portfolio/tabla-de-analisis-de-sentenciasde-la-corte-suprema-de-justicia-sobre-baldios-2014-2016/
} 
Mediante este análisis sostenemos que la discusión en el ámbito constitucional de la acción de tutela, sobre la propiedad de baldíos de la nación ha conducido a una disputa y tensión entre una teoría basada en derechos de la propiedad privada asociada a la jurisprudencia de la Corte Suprema de Justicia y una teoría utilitarista de la propiedad sostenida por la Corte Constitucional.

El texto está organizado de la siguiente manera: en la sección que sigue a esta introducción, se explica la teoría utilitarista y la teoría basada en derechos de Locke. Posteriormente, se presentan las providencias de la Corte Suprema y de la Corte Constitucional, enfocándonos en la relación entre los argumentos y consideraciones expuestas en esas decisiones y los postulados de las teorías explicadas. Finalmente, se expondrán algunas conclusiones.

\section{De Bentham a Locke: utilitarismo y teoría basada en derechos}

Alexander y Peñalver publicaron en 2012: An Introduction to Property Theory. El texto sintetizaba las principales teorías históricas que se han propuesto sobre los derechos de propiedad y se apoyaba en diversas fuentes secundarias para que esa síntesis abordara con suficiencia cada teoría a un nivel introductorio.

La serie de teorías que identificaron fueron: las teorías utilitaristas de la propiedad, las teorías de Locke y los libertarios, la teoría hegeliana de la propiedad, la teoría kantiana de la propiedad y la teoría de la prosperidad humana. Según los autores, las dos primeras han tenido la mayor influencia en el derecho de propiedad estadounidense (Alexander \& Peñalver, 2012) y, como veremos, estas dos teorías también pueden relacionarse con la discusión sobre la propiedad baldía en Colombia.

Por teorías de propiedad entendemos lo expuesto por Alexander \& Peñalver (2012):

(...) si definimos la propiedad como una categoría de doctrina jurídica referida a la asignación de derechos a recursos materiales, podemos entender una teoría de la propiedad como un intento para proveer una justificación normativa para asignar esos derechos en una forma particular. Al nivel más básico, una teoría de la propiedad podría responder la pregunta de cuáles intereses humanos son relevantes para el proyecto de asignar derechos de propiedad. (p. 6)

Las teorías utilitaristas, en cuanto consecuencialistas, evalúan moralmente a las reglas y a las instituciones si proveen buenas o malas consecuencias (Smart \& Williams, 1973). En la tarea de evaluar la bondad o maldad de una consecuencia el criterio fundamental es que maximicen la utilidad o el bienestar (Sen \& Williams, 1982). Frente a la utilidad, el utilitarismo sostiene que es el individuo quien define sus preferencias (su función de utilidad) y una elección socialmente considerada debía agregar los cálculos que cada individuo realizaba respecto de la satisfacción de sus preferencias. Bentham (1781), uno de los grandes exponentes del utilitarismo, 
propone que esa agregación se realice mediante la suma neta del placer individual de todas las personas afectadas por la decisión. Todas estas consideraciones a la hora de una teoría utilitarista de la propiedad conducen a lo siguiente:

Una teoría utilitarista de los derechos de propiedad es aquella que propone aquel sistema que brinde la mayor utilidad neta agregada o en otros términos maximice la riqueza general neta ${ }^{3}$. Los derechos de propiedad son un medio para un bien básico mayor que es la utilidad o el bienestar. (Alexander \& Peñalver, 2012, p. 17)

Por su parte, la teoría de Locke sobre la propiedad solo cumple un papel secundario en su propósito general de proponer una fundamentación del Estado. En cuanto a la propiedad, tiene una fuerte influencia del derecho natural racionalista (Alexander \& Peñalver, 2012; Waldron, 1990), esto es, que los seres humanos conocen el contenido de sus derechos y obligaciones mediante sus facultades racionales y aquellos son anteriores a cualquier organización social establecida. Todo ser humano tiene el derecho y el deber de preservarse y sobrevivir, y en cumplimiento de este deber todos tienen el mismo derecho a usar las cosas y adoptarlas como medios para proveerse esa subsistencia (Alexander \& Peñalver, 2012; Laslett, 1988). Los arreglos del derecho positivo deben dirigirse a preservar este derecho. En ese sentido debe recordarse que el trabajo que los individuos le imprimen a las cosas es el criterio central del fundamento de derechos de propiedad privada sobre los bienes en la postura de Locke (Alexander \& Peñalver, 2012; Laslett, 1988). En algunas aproximaciones, este principio de la propiedad basada en el trabajo se asemeja al derecho de propiedad que tiene un creador sobre las cosas que concibe intencionalmente (Tully, 1980) ${ }^{4}$.

Pese a la relativa antigüedad de las propuestas teóricas explicadas, el debate sobre la posibilidad y los modos de apropiarse de los terrenos baldíos en Colombia demuestra la vigencia y pertinencia de conocer en detalle estas teorías sobre la propiedad. Y precisamente las posturas adoptadas por la Corte Constitucional y la Corte Suprema de Justicia mostrarán que la discusión jurisprudencial actual puede enmarcarse en la tensión entre ambas teorías de los derechos de propiedad, como pasará a mostrarse a continuación.

\section{Bentham y Locke en nuestras Cortes}

De acuerdo con el Código Civil, un baldío es un bien de la Unión situado dentro de los límites territoriales que carece de otro dueño ${ }^{5}$. En otras palabras, aquello que no sea de un particular y esté dentro de los límites del territorio nacional es un baldío. Por su parte la Ley 160 de 1994, en su artículo 65 establece que "la propiedad de los terrenos baldíos adjudicables, sólo puede adquirirse mediante título traslaticio de dominio otorgado por el Estado a través del Instituto Colombiano de la Reforma

\footnotetext{
${ }^{3}$ Acerca de una distinción relevante entre el principio de maximización de la utilidad y el utilitarismo puede consultarse Posner (1979).

${ }^{4}$ Estas aproximaciones discuten algunas objeciones formuladas por Nozick (1974), acerca del principio del trabajo como fundamento de la apropiación privada.

${ }^{5}$ Artículo 675 del Código Civil.
} 
Agraria, o por las entidades públicas en las que delegue esta facultad" (Ley 160, 1994, art. 65). Sin embargo, la Ley 200 de 1936 establece una presunción legal a favor de los colonos que exploten económicamente el fundo ${ }^{6}$.

Como se dijo en la introducción, buscamos, a partir del análisis de las normas mencionadas y de la interpretación que de ellas hacen en distintas sentencias de carácter civil o constitucional, identificar las teorías sobre el derecho de propiedad que están involucradas en las decisiones de las altas Cortes. Esto con el fin de determinar los cambios que conceptualmente ha experimentado el concepto de propiedad.

El punto de partida de la discusión podemos encontrarlo en la Sentencia T-488 de 2014 en la que la Corte Constitucional determina que, en juicios de pertenencia de bienes inmuebles, no basta comprobar los requisitos de posesión para declarar la adquisición de un bien por prescripción pues es necesario determinar si el bien es un fundo privado o, por el contrario, es un bien baldío. Por otra parte, la Corte advierte que es el juez, en este caso el Juez Promiscuo del Circuito de Orocué (Casanare) quien debe determinar la naturaleza del bien en los procesos de pertenencia con el fin de establecer si se trata de un bien susceptible de adquirirse por prescripción. Para este efecto, debe contar con una prueba fundamental: el concepto del INCODER acerca de la calidad del bien como requisito sine qua non para dar inicio al proceso. Para la Corte indicios como la ausencia de propiedad inscrita $^{7}$ o de cadenas traslaticias del derecho de dominio que den fe del dominio privado y que la solicitud de prescripción se dirija contra personas indeterminadas conducen a la convicción de que se estaba ante un predio baldío.

Algunas de las consideraciones vertidas en este fallo encuadran a la decisión dentro de la teoría utilitarista de los derechos de propiedad. La Corte inició recordando que:

Aunque la prescripción o usucapión es uno de los modos de adquirir el dominio de los bienes corporales, raíces o muebles que están en el comercio, los terrenos baldíos obedecen a una lógica jurídica y filosófica distinta, razón por la cual estos tienen un régimen especial

\footnotetext{
${ }^{6}$ Artículo 1: Se presume que no son baldíos, sino de propiedad privada, los fundos poseídos por particulares, entendiéndose que dicha posesión consiste en la explotación económica del suelo por medio de hechos positivos propios de dueño, como las plantaciones o sementeras, la ocupación con ganados y otros de igual significación económica. El cerramiento y la construcción de edificios no constituyen por sí solos pruebas de explotación económica pero sí pueden considerarse como elementos complementarios de ella. La presunción que establece este artículo se extiende también a las porciones incultas cuya existencia se demuestre como necesaria para la explotación económica del predio, o como complemento para el mejor aprovechamiento de éste, aunque en los terrenos de que se trate no haya continuidad o para el ensanche de la misma explotación. Tales porciones pueden ser conjuntamente hasta una extensión igual a la mitad de la explotada y se reputan poseídas conforme a este artículo (reformado por el artículo 2 de la ley 4 de 1973).

${ }^{7}$ El que no exista un propietario inscrito, o que no haya matrícula inmobiliaria es un elemento que puede determinar la naturaleza del predio. Sin embargo, es necesario recordar que en el art. 57 de la Ley 1579 de 2012 se ordena la apertura de la matrícula inmobiliaria que identifique a los predios baldíos a nombre de la nación. Independientemente de que se realice o no tal registro inmobiliario, el propietario de los baldíos es la nación (art. 675 C.C. y Ley 1579 de 2012). En este orden, no solo la no existencia de matrícula hace pensar que el predio sea baldío.
} 
que difiere del consagrado en el Código Civil. (Corte Constitucional 2014)

Y luego de recordar que los postulados entre la usucapión y la adjudicación previa ocupación obedece a supuestos filosóficos distintos, sostiene que:

Esta limitación en el comercio de los baldíos tampoco quebranta la igualdad en relación con los bienes privados, sobre los cuales sí procede la prescripción adquisitiva, por cuanto 'quien posee un bien fiscal, sin ser su dueño, no está en la misma situación en que estaría si el bien fuera de propiedad de un particular. En el primer caso su interés particular se enfrenta a los intereses generales, a los intereses de la comunidad; en el segundo, el conflicto de intereses se da entre dos particulares.

$(\ldots)$

El trato diferenciado sobre los terrenos baldíos que se refleja, entre otros aspectos, en un estatuto especial (Ley 160 de 1994), en la prohibición de llevar a cabo procesos de pertenencia y en la consagración de requisitos para ser beneficiarios del proceso de adjudicación administrativa, responde a los intereses generales y superlativos que subyacen.

\section{$(\ldots)$}

Lo dicho hasta el momento no implica que la dignificación del trabajador agrario deba realizarse a costa del interés general y el desarrollo del país. Por el contrario, el acceso a la propiedad a quienes carecen de ella, contribuye por esa vía al mejoramiento de toda la sociedad. Propósito que la Ley 160 de 1994 retoma al establecer que el primer objetivo de la reforma agraria es promover y consolidar la paz, a través de mecanismos encaminados a lograr la justicia social y la democracia participativa. (Corte Constitucional 2014)

La Corte entonces considera que en el acceso a la propiedad baldía están involucrados los intereses generales y de la comunidad, y que las restricciones para reconocer derechos de propiedad a particulares sobre este tipo de bienes deben responder a intereses de maximización del bienestar social y no simplemente de reconocer los intereses individuales que "en este caso" estaban representados en la persona que aspiraba a ser reconocida como propietaria.

Ese mismo año la Sala de Casación Civil de la Corte Suprema de Justicia conocería en segunda instancia de la acción de tutela promovida por una procuradora ambiental y agraria en la que solicita la nulidad de todo un proceso de pertenencia al no haberse vinculado ni a la Procuraduría, ni al INCODER. La Corte Suprema adoptó reglas similares a las de la Corte Constitucional en el fallo arriba mencionado: en los procesos de pertenencia es necesario determinar la naturaleza del bien en discusión. Cuando en un proceso de pertenencia el bien en discusión carece de 
historia inmobiliaria es necesario que el juez decrete pruebas adicionales con el fin de determinar que el mismo puede ser objeto de este tipo de procesos. En los procesos de pertenencia se debe vincular al INCODER, pues este es el encargado de desvirtuar la presunción del art. 1 de la Ley 200 de $1936^{8}$. Por otra parte, la Corte consideró que son indicios que permiten inferir que un bien es baldío la ausencia de antecedentes registrales, de titulares de derechos reales sobre el predio o de titulares inscritos.

La Corte Suprema citó expresamente el fallo de la Corte Constitucional y utilizó argumentos utilitaristas similares a los de esta última. Expuso que, sin las precauciones probatorias, el juez de la pertenencia había adjudicado ilegítimamente "un bien presuntamente baldío", que resulta en la afectación del "interés público y la correcta administración de justicia, por lo que se torna necesario estudiar de fondo la procedencia del amparo, en aras de proteger el patrimonio del Estado" (Corte Suprema de Justicia, STC16151-2014).

El fallo anterior sería reconocido como precedente en un fallo posterior de noviembre de 2014 en el que la Corte Suprema vuelve a concederle la tutela al INCODER instaurada contra los juzgados promiscuos del circuito y de familia de Paz de Ariporo (Casanare) y vuelve a citar como precedente relevante la Sentencia T-488 de 2014 y en el que se considera la misma necesidad de protección del patrimonio público haciendo inviables procesos de pertenencia sobre tierras baldías.

Hasta este momento las decisiones de la Corte Suprema de Justicia habían acogido el criterio expuesto en la Sentencia T-488 de 2014, señalando reglas similares de participación del INCODER dentro de los procesos de pertenencia adelantados sobre inmuebles presuntamente baldíos. También coincidían en el criterio utilitarista de que el procedimiento administrativo de adjudicación era el adecuado para salvaguardar el interés público. Esas decisiones también avalaban a la acción de tutela como mecanismo idóneo y procedente para que el INCODER invalidara los fallos que declaraban la prescripción, lo que permitía que la jurisdicción constitucional conociera y se involucrara en un proceso de naturaleza civil como es la prescripción.

Sin embargo, esto cambió con el fallo de la Corte Suprema de Justicia STC12702015 proferido en febrero de 2015, en el que se niega la procedencia de la tutela interpuesta por el INCODER, considerando que cuenta con el recurso extraordinario de revisión para hacer valer su participación dentro de procesos de pertenencia sobre predios presuntamente baldíos. Así, la Corte da el mensaje que es la jurisdicción civil la que está Ilamada a fallar sobre el tema de la pertenencia y, así mismo es un intento de aislar el asunto del ámbito constitucional ${ }^{9}$. Al considerar

\footnotetext{
${ }^{8}$ M.P.: Ariel Salazar Ramírez, Sala de Casación Civil, STC15027-2014, 4/11/2014.

${ }^{9}$ M.P.: Jesús Vall de Rutén Ruíz, Sala de Casación Civil
} 
improcedente la tutela, la Corte no involucra argumentos o consideraciones sobre el fondo del asunto; es decir, sobre alguna posición doctrinaria acerca de un criterio utilitarista o basada en derechos sobre los derechos de propiedad. Otro aspecto por resaltar es que la reacción a la jurisprudencia anterior solo tomó un lapso de siete meses desde que había sido proferida la Sentencia T-488 de 2014.

Solo un mes después, en marzo de 2015 la Corte Suprema variaría la decisión respecto del fallo arriba mencionado, es decir, consideraría procedente la acción de tutela interpuesta por el INCODER para invalidar decisiones judiciales de pertenencia sobre predios presuntamente baldíos. Y aquí se exponen nuevamente consideraciones utilitaristas similares en casos anteriores de la necesidad de preservar el régimen especial de los baldíos (su imprescriptibilidad), en aras de proteger el patrimonio público y la destinación específica de los baldíos a garantizar el bienestar general. Este criterio sería sostenido en decisiones similares del 17 de marzo, 6 de abril; 5 y 11 de agosto; 6 y 30 de septiembre; 28 de octubre; y 25 de noviembre de $2015^{10}$.

En febrero de 2016 la Corte Suprema hace un cambio en su posición frente a los juicios de pertenencia y niega la tutela al INCODER. Lo más interesante de este fallo son las razones y reglas que introduce la Corte: en primer lugar, la Corte señala que la tutela no es el medio idóneo para que el INCODER impugne una sentencia de un proceso en el que considera que debió ser vinculado. Para estos eventos, señala, el INCODER cuenta con el recurso de revisión. En segundo lugar, la Corte Ilama la atención que, según las presunciones de la Ley 200 de 1936 y el Código Civil, el predio explotado económicamente se presume de propiedad privada y sí el Estado quiere desmentir la anterior presunción, deberá acudir a la presunción contraria: que el predio no ha sido explotado económicamente y que es, por lo tanto, baldío. En tercer lugar, la Corte afirma, contrariamente a lo dicho por ella misma en los dos años anteriores, que el certificado expedido por la oficina de registro de instrumentos públicos para los procesos de pertenencia, tiene la única finalidad de identificar a los legítimos contradictores de la pretensión y no es un elemento para indicar la naturaleza jurídica del predio ${ }^{11}$.

Esta decisión es, en primer lugar, un nuevo intento de clausurar la discusión del asunto en el ámbito constitucional, al insistir en que el recurso de revisión es el mecanismo idóneo para revisar lo decidido en juicios de pertenencia sobre presuntos baldíos y entonces las acciones de tutela que interpone el INCODER con ese propósito no son procedentes. Pero en segundo lugar, la interpretación

\footnotetext{
${ }^{10}$ Sala de Casación Civil STC2973-2015, M.P.: Jesús Vall de Rutén Ruiz; Sala de Casación Civil STC3765-2015, Jesús Vall de Rutén Ruiz; Sala de Casación Civil. STC10474-2015, M.P. Luis Armando Tolosa Villabona; Sala de Casación Civil. STC10720-2015, M.P. Álvaro Fernando García Restrepo; Sala de Casación Civil. STC11637-2015, M.P. Luis Armando Tolosa Villabona; Sala de Casación Civil. STC13435-2015, M.P. Fernando Giraldo Gutiérrez; Sala de Casación Civil. STC14853-2015, M.P. Ariel Salazar Ramírez y Sala de Casación Civil. STC16320-2015, M.P. Margarita Cabello Blanco.

${ }^{11}$ M.P.: Luis Armando Tolosa Villabona Sala de Casación Civil. STC1776-2016 16/02/2016.
} 
y uso que hace de las presunciones de la Ley 200 de 1936 involucran una teoría de la propiedad distinta a la del utilitarismo que venía siendo alegada en la jurisprudencia. Al contrario, la referencia a la importancia de la explotación económica al trabajo que se imprime sobre la tierra será el criterio determinante para acceder a la propiedad; pero esta propiedad será adquirida sin atención a consideraciones generales de maximización del bienestar social. De hecho, la incapacidad para determinar la naturaleza baldía de los bienes solicitados en restitución hace inoperantes las restricciones y limitaciones de la extensión de la adjudicación que se imponen para titular administrativamente la propiedad baldía. Esta decisión supone entonces otro sistema de derecho de propiedad, otra teoría de los derechos de propiedad distinta a la de la Corte Constitucional.

Tal criterio y modificación jurisprudencial son ratificados en sentencia de abril del mismo año citando la Sentencia STC1776-2016 del 16 de febrero de 2016 (M.P. Luis Armando Tolosa), como precedente; y al reiterar la decisión reivindica la importancia de la explotación para reconocer y derivar derechos de propiedad privada sobre la tierra, en sintonía con la tesis de Locke sobre el fundamento para la propiedad privada. Lo importante de los dos fallos anteriores es que parten de la base de que es la explotación económica, y no la historia catastral del predio, la que determina la naturaleza privada, y es obligación del INCODER desvirtuar esta presunción.

Este cambio de jurisprudencia no fue sostenido. En sentencia de agosto de 2016, al confirmar la sentencia del Tribunal de Yopal la Corte advierte que:

Solamente el Estado tiene el poder de transferir los bienes baldíos a favor de los particulares por medio de la adjudicación y con el cumplimiento de ciertos requisitos, es más, en el trascurso de los años el legislador ha prohibido la adquisición de esos bienes por otro modo distinto a ese, ni siquiera por usucapión, así por ejemplo el artículo 2519 del Código Civil establece que 'Los bienes de uso público no se prescriben en ningún caso $^{\prime 12}$

Por otra parte, se afirma que la carga probatoria es del demandante, es decir, siempre debe demostrar la naturaleza del predio, ya sea para que este le sea adjudicado por vía administrativa o para que declare en su cabeza la usucapión por vía judicial; y en los casos que se pueda colegir que el predio es baldío por ausencia de dueños y registro, el juez debe decretar pruebas tendientes a definir la naturaleza de este. Finalmente, la Corte reafirma lo establecido en el artículo 65 de la Ley 180 de 1994: la única forma de adquirir bienes baldíos es a través de la adjudicación por parte del Estado. La procedibilidad del amparo de tutela constitucionalizó de nuevo la cuestión y fue respuesta inmediata a la tesis de la revisión como mecanismo idóneo para ventilar el asunto.

${ }^{12}$ Ver en el artículo 2519 del Código Civil Colombiano disponible en http://www.amb.gov.co/jdownloads/Documentos/ Administracin\%20y\%20Financiera/Codigo_civil.pdf 
Finalmente, la Corte Constitucional, en Sentencia T-461 de 2016 reitera el criterio y las consideraciones de la T-488 de 2014, especialmente, en su criterio utilitarista de que el sistema de adjudicación de baldíos existente propende por maximizar el bienestar social y responder a los intereses generales antes que el otorgamiento y reconocimiento de derechos de propiedad privada sobre la tierra, sin restricciones y limitaciones de extensión mediante el procedimiento judicial de prescripción. Recuerda la Corte que la única forma de adquirir bienes baldíos es a través de la adjudicación por parte del Estado y que la mera ocupación del bien no es suficiente como sí lo es para adquirir los bienes privados. Y avala el hecho de que el INCODER no cuenta con un mecanismo distinto a la acción de tutela para ventilar de nuevo los asuntos objeto de decisiones de prescripción, luego preserva la posibilidad de que el asunto siga siendo constitucionalizado.

\section{Conclusiones}

Los aportes de Alexander \& Peñalver sobre las teorías del utilitarismo y de Locke que analizan la propiedad en el derecho estadounidense, no son tan ajenos al contexto colombiano en el que las consideraciones sobre los sistemas para adjudicar derechos de propiedad privada sobre terrenos baldíos, se asocian con los argumentos defendidos por las Cortes Constitucional y Suprema de Justicia.

La revisión del conjunto de fallos proferidos por la Corte Constitucional y la Corte Suprema de Justicia en los que el centro del debate es determinar la naturaleza baldía o privada de los bienes inmersos en procesos judiciales de prescripción, muestran una cierta estabilidad en el periodo estudiado de la tesis esgrimida por la Corte Constitucional y cuyos supuestos pudimos asociar a las teorías utilitaristas de la propiedad. El principal elemento de contacto entre la tesis de la Corte Constitucional y la teoría utilitarista se refiere al criterio de maximización del bienestar que está implícito en sus consideraciones. De ese modo, el Tribunal Constitucional destacó la importancia de garantizar la imprescriptibilidad de los bienes baldíos con fundamento en que están afectados a una destinación social específica que reporta mayor bienestar social.

Solo hasta febrero de 2016 la teoría sería desafiada desde la Corte Suprema de Justicia por una postura asociada a las tesis de Locke y a la importancia del trabajo y la explotación económica como criterio definitivo para asignar derechos de propiedad y, con ello, desvirtuar el carácter baldío de un terreno.

Este análisis jurisprudencial a partir de dos teorías específicas de la propiedad también abre líneas de investigación en dos aspectos: (i) la resistencia a la constitucionalización de ciertos conceptos propios del derecho privado y (ii) la consistencia de las tesis de las Cortes con teorías de la propiedad en el derecho continental. Acerca del primer aspecto, la disputa evidenciada en los fallos estudiados muestra algunos mecanismos para conservar la discusión sobre la propiedad en el ámbito de la jurisdicción civil, mediante la improcedencia de acciones de tutela y la insistencia en llevar estos procesos mediante el recurso de revisión ante la misma Corte Suprema de Justicia. Sin embargo, la decisión más 
reciente al respecto preserva la discusión constitucional sobre los baldíos y de los modos de asignación de derechos de propiedad atendiendo, a criterios más amplios que el trabajo individual, como hacer efectiva la restricción a la acumulación de predios baldíos y la administración sobre las tierras baldías en general.

Acerca del segundo aspecto, las teorías de la propiedad involucradas en las decisiones judiciales, pueden ser un enfoque útil para realizar trabajos sistemáticos sobre el derecho de propiedad en Colombia al tiempo que dialogan con los esfuerzos hechos para periodos y ámbitos institucionales distintos a los de la labor judicial. Este es un punto de partida de una agenda de investigación que sin duda aún está por explorar.

\section{Referencias bibliográficas}

Alexander, G.S. \& Peñalver, E.M. (2012). An Introduction to Property Theory. Cambridge: Cambridge University Press.

Bentham, J. (1781). An Introduction to the Principles of Morals and Legislation.

Céspedes-Báez, L., Peña-Huertas, R. d., Cabana-González, D.S. y Zuleta-Ríos, S. (2015). Who Owns the Land? Litigants, Justices, Colonos, and Titleholders' Struggle to Define the Origins of Private Property in Colombia, Global Jurist, 329-459.

Corte Constitucional de Colombia. Sentencia T-488 de 2014. Magistrado Ponente: Jorge Iván Palacio Palacio.

Corte Constitucional de Colombia. Sentencia T-461 de 2016. Magistrado Ponente: Jorge Iván Palacio Palacio.

Corte Suprema de Justicia. Sala de Casación Civil. STC15027-2014. Magistrado Ponente: Ariel Salazar Ramírez.

Corte Suprema de Justicia. Sala de Casación Civil. STC16151-2014. Magistrado Ponente: Fernando Giraldo Gutiérrez.

Corte Suprema de Justicia. Sala de Casación Civil. STC1270-2015: Magistrado Ponente: Jesús Valle de Rutén Ruiz.

Corte Suprema de Justicia. Sala de Casación Civil. STC2628-2015. Magistrado Ponente: Luis Armando Tolosa Villabona.

Corte Suprema de Justicia. Sala de Casación Civil. STC2973-2015. Magistrado Ponente: Luis Armando Tolosa Villabona.

Corte Suprema de Justicia. Sala de Casación Civil. STC3765-2015. Magistrado Ponente: Jesús Vall de Rutén Ruiz.

Corte Suprema de Justicia. Sala de Casación Civil. STC10474-2015. Magistrado Ponente: Luis Armando Tolosa Villabona.

Corte Suprema de Justicia. Sala de Casación Civil. STC10720-2015. Magistrado Ponente: Álvaro García Restrepo.

Corte Suprema de Justicia. Sala de Casación Civil. STC11637-2015. Magistrado Ponente: Luis Armando Tolosa Villabona.

Corte Suprema de Justicia. Sala de Casación Civil. STC13435-2015. Magistrado Ponente: Fernando Giraldo Gutiérrez. 
Corte Suprema de Justicia. Sala de Casación Civil. STC14853-2015. Magistrado Ponente: Ariel Salazar Ramírez.

Corte Suprema de Justicia. Sala de Casación Civil. STC16320-2015. Magistrado Ponente: Margarita Cabello Blanco.

Corte Suprema de Justicia. Sala de Casación Civil. STC1776-2016. Magistrado Ponente: Luis Armando Tolosa Villabona.

Corte Suprema de Justicia. Sala de Casación Civil. STC5201-2016. Magistrado Ponente: Luis Armando Tolosa Villabona.

Corte Suprema de Justicia. Sala de Casación Civil. STC11024-2016. Magistrado Ponente: Álvaro Fernando García Restrepo.

J.J.C. \& Williams, B. (1973). Utilitarianism: For and Against. Cambridge: Cambridge University Press. Laslett, P. (1988). John Locke, Two Treatises of Government. Cambridge: Cambridge University Press.

Nozick, R. (1974). Anarchy, State, and Utopia. New York: Basic Books.

Posner, R. (1979). Utilitarianism, Economics, and Legal Theory. The Journal of Legal Studies, 103-140.

Quinche-Ramírez, M.F., Peña-Huertas, R.D.P., Parada-Hernández, M.M., Ruiz- González, L.E. y ÁlvarezMorales, R. (2015). El amparo de tierras: La acción, el proceso y el juez de restitución. Bogotá: Editorial Universidad del Rosario.

Rengifo-Gardeazábal, M. (2011). Teoría general de la propiedad. Bases para la construcción de un derecho civil realista en el ámbito de la tradición jurídica romana. Bogotá: Ediciones UniAndes.

Sen, A. \& Williams, B. (eds.) (1982). Utilitarianism and Beyond. Cambridge: Cambridge University Press Smart.

Tully, J. (1980). A Discourse on Property. New York: Cambridge University Press.

Waldron, J. (1990). The Right to Private Property. Oxford: Clarendon Press.

\section{Anexo $1^{13}$}

\footnotetext{
${ }^{13}$ Véase: Cuadro de Análisis de las sentencias revisadas sobre baldíos (2014-2016) http://www.observatoriodetierras.org/portfolio/tabla-de-analisis-de-sentencias-de-la-corte-suprema-de-justicia-sobrebaldios-2014-2016/
} 